\title{
Determining the verse of magnetic turbulent cascades in the Earth's magnetospheric cusp via transfer entropy analysis: preliminary results
}

\author{
M. Materassi ${ }^{1}$, A. Wernik ${ }^{2}$, and E. Yordanova ${ }^{3}$ \\ ${ }^{1}$ Istituto dei Sistemi Complessi del CNR, Florence, Italy \\ ${ }^{2}$ Space Research Center of the Polish Academy of Science, Warsaw, Poland \\ ${ }^{3}$ Space Research Institute of the Bulgarian Academy of Science, Sofia, Bulgaria
}

Received: 5 September 2005 - Revised: 5 March 2007 - Accepted: 4 April 2007 - Published: 16 April 2007

\begin{abstract}
The inter-scale coupling in the dynamics of the magnetic field in the Earth's magnetospheric cusp is studied with the technique of transfer entropy. This is a non-linear data analysis technique conceived to determine which is the process that plays the role of the "dynamical driver" between two processes interacting.

The time series of the magnetic field components measured along the trajectory of a spacecraft through the cusp are decomposed via continuous wavelets, so a time series of the square modulus of the wavelet coefficients may be associated to each scale $\ell$ considered. The coupling between to two nearby scales is studied, with the purpose of singling out turbulent cascade directions from large to small scales and viceversa.

Preliminary physical conclusions are proposed.
\end{abstract}

\section{Introduction}

In fluid as well as in magneto-hydrodynamic turbulence, the presence of an inter-scale dynamics shows up clearly Frisch (1995): excitations on many different space scales interact with each other. Non-linear terms in the equations governing turbulent systems are responsible for the inter-scale dynamics. For instance, in the fluid case three-wave interaction and cascades are all phenomena governed by such non-linear terms in the Navier-Stokes equations.

This paper is devoted to the study of the inter-scale dynamics of the turbulent magnetic field in the Magnetosphere of the Earth, in particular to the study of the verse of cascade processes taking place in the turbulent magnetic field in the magnetospheric cusp (MC).

The traditional scenario of fluid turbulence predicts that larger scale excitations give rise to smaller ones via fragmen-

Correspondence to: M. Materassi

(massimo.materassi@fi.isc.cnr.it) tation, which is referred to as direct cascade Frisch (1995). The opposite phenomenon, the inverse cascade, in which structures aggregate giving rise to larger scale eddies, seems to be confined to theoretically well defined situations, as the 2-D turbulence described in Kraichmann and Montgomery (1980). However, there are experimental indications of direct and inverse cascade processes in the magnetic field of the magnetospheric turbulent boundary layer, as Savin and his co-Authors showed in Savin et al. (2004). This motivated the present research, in which a new data analysis technique, introduced in Schreiber (2000) and refined in Keiser and Schreiber (2002), here referred to as transfer entropy analysis (TEA), is adapted to study the "cascades" in the magnetospheric turbulence.

The TEA is based on the principle that interacting processes exchange information entropy Haken (1983): given two interacting processes $X$ and $Y$, the transfer entropy $T_{X \rightarrow Y}$ from $X$ to $Y$ will measure the influence of $X$ on $Y$, so that making a comparison between the quantities $T_{X \rightarrow Y}$ and $T_{Y \rightarrow X}$ one should be able to say which is the process more strongly influencing the other.

The TEA may be exploited to determine in which sense the turbulent cascade evolves, whether direct or inverse. Indeed, assume to deal with a time series $s(t)$ describing a turbulent evolution via measurements made by a probe moving very slowly through the turbulent flux, so that some definite (probably unknown) relationship exists between the time scales in $s(t)$ and the space scales in the field theory. Suppose to be able to extract a collection of time series $\varepsilon_{\ell}(t)$ describing how $s(t)$ appears at each time scale $\ell$. Then, apply the TEA to couples of time series $\varepsilon_{\ell}(t)$ and $\varepsilon_{\ell^{\prime}}(t)$ with $\ell>\ell^{\prime}$ : in this case, if the TEA indicates that $\varepsilon_{\ell^{\prime}}$ influences $\varepsilon_{\ell}$, a diagnosis of direct cascade may be done, while if the smaller scale evolution $\varepsilon_{\ell}$ influences the larger scale evolution $\varepsilon_{\ell^{\prime}}$, one might state to be facing an inverse cascade process.

Actually, more complex mechanisms could be invoked to explain turbulent fluctuations in a plasma, as those described

Published by Copernicus GmbH on behalf of the European Geosciences Union and the American Geophysical Union. 


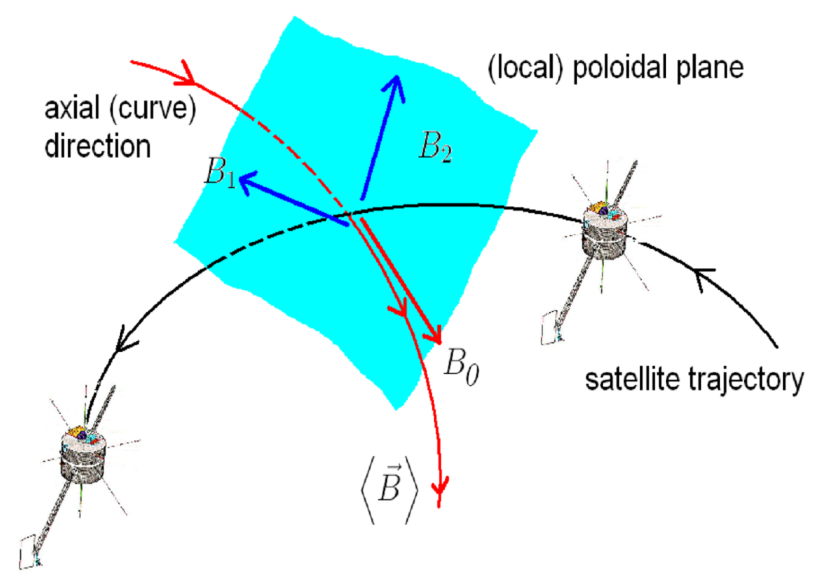

Fig. 1. A pictorial vision of the axial-poloidal decomposition of the vector $\boldsymbol{B}$ as measured by the POLAR satellite along its trajectory.

Strong bulk field,

more intermittent transverse fluctuations

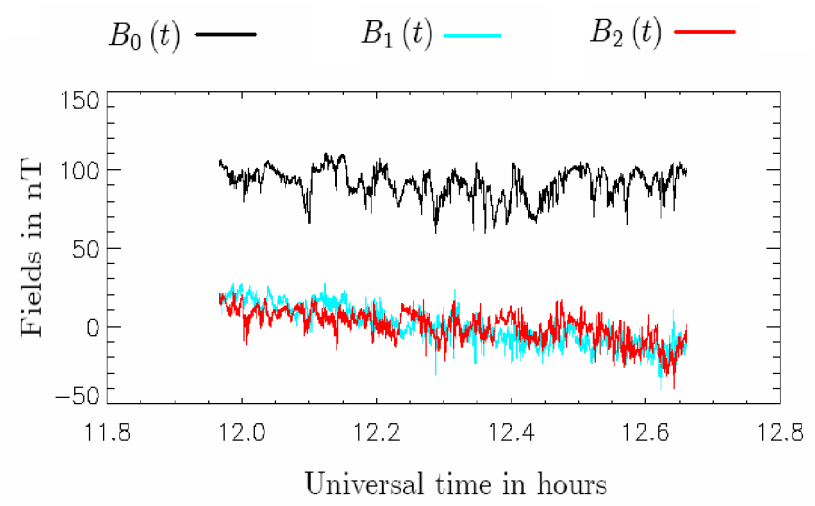

Fig. 2. The three components of the vector $\boldsymbol{B}$ as measured by the POLAR satellite along its trajectory.

in Chang et al. (2004) for the dynamics of structures arising from resonances. In those scenarios no definte verse in the cascades appear, one rather speaks about multi-scale coherent structures in interaction: confuse pictures about the verse of cascades emerging from the TEA could support those richer scenarios (which seems to be the case, indeed, in these preliminary results).

The data used here are magnetic field measurements collected by the instruments on board of the NASA POLAR spacecraft Russel et al. (1995). The time series considered is that of the three components of $\boldsymbol{B}$ measured in the orbit segment starting at 11:55 UT and ending at 12:44 UT, on 11 April 1997. Measurements were taken in the northern cusp region in the magnetic latitudes range $59.87^{\circ}-67.95^{\circ}$, and from 13:40 to 14:17 MLT. These time series have been elaborated also in Yordanova et al. (2005). If the trajectory of the spacecraft is $\boldsymbol{x}_{S}(t)$ then the time series at hand are related to the local fields as

$\boldsymbol{B}(t)=\boldsymbol{B}\left(\boldsymbol{x}_{S}(t), t\right)$.

The time series analysed are described in some detail in Yordanova et al. (2005) and Yordanova et al. (2004). As described in Yordanova et al. (2005), these magnetic fluctuations are anisotropic, since the turbulent statistical and spectral features along the average field $\langle\boldsymbol{B}\rangle$ (axial direction) and perpendicular to it (poloidal plane) are different. Expecting this anisotropy to lead to different cascade behaviours in the axial and poloidal directions, here we apply the TEA separately to the time series of the axial component

$B_{0}=\frac{\boldsymbol{B} \cdot\langle\boldsymbol{B}\rangle}{|\langle\boldsymbol{B}\rangle|}$

and to the ones of the two poloidal components indicated as $B_{1}$ and $B_{2}$, so that the total field reads

$\left\{\begin{array}{l}\boldsymbol{B}(t)=B_{0}(t) \hat{e}_{/ /}+B_{1}(t) \hat{e}_{\perp 1}+B_{2}(t) \hat{e}_{\perp 2}, \\ \hat{e}_{/ /}=\frac{\langle\boldsymbol{B}\rangle}{|\langle\boldsymbol{B}\rangle|},\langle\boldsymbol{B}\rangle \cdot \hat{e}_{\perp 1}=0,\langle\boldsymbol{B}\rangle \cdot \hat{e}_{\perp 2}=0, \\ \hat{e}_{\perp 1} \cdot \hat{e}_{\perp 2}=0 .\end{array}\right.$

The axial-poloidal decomposition is illustrated in Fig. 1. Note that $\langle\boldsymbol{B}\rangle$ is in principle an ensamble average and might depend on time for nonstationary evolutions. In practice, this will be calculated as the time average of $\boldsymbol{B}$ instead, hence will be a constant vector. In all our calculations, we assume the equivalence of ensemble and time statistics (stationarity) and space and time statistics (Taylor hypothesis) Frisch (1995). Plausibility reasons for accepting this hypotheses in the same data segments usede here were given in Yordanova et al. (2004).

In Fig. 2 the three time series $B_{0}(t), B_{1}(t)$ and $B_{2}(t)$ are depicted. It is visible how

$\left\langle B_{i}\right\rangle \ll\left\langle B_{0}\right\rangle$.

At a glance, the poloidal components appear to be more intermittent than $B_{0}$.

\section{Transfer entropy and the magnetic turbulence}

The transfer entropy from a process $Y$ to a process $X$ in the time lapse $\tau$ is the quantity of information that the state of $X$ has at the time $t+\tau$ due only to the state of $Y$ at the time $t$ Schreiber (2000). If the processes $X$ represented by $x(t)$ and $Y$ represented by $y(t)$ are treated as probabilistic evolutions, the transfer entropy representing the dynamical influence of the process $Y$ on the process $X$ is defined as

$$
\begin{aligned}
& T_{Y \rightarrow X}(\tau)= \\
& =\sum_{\substack{x(t) \\
x(t-\tau) \\
y(t-\tau)}} p(x(t+\tau), x(t), y(t)) \log _{2}\left(\frac{p(x(t+\tau) \mid x(t), y(t))}{p(x(t+\tau) \mid x(t))}\right),
\end{aligned}
$$


where $p(x(t+\tau), x(t), y(t))$ is the joint probability of having the states $x(t)$ and $y(t)$ at the time $t$ and $x(t+\tau)$ at time $t+\tau$, while $p(x(t+\tau) \mid x(t), y(t \tau))$ and $p(x(t+\tau) \mid x(t))$ are conditional probabilities. In the definition (5) the delay $\tau$ is the only time parameter on which $T_{Y \rightarrow X}$ depends if the processes are stationary.

The comparison between the two quantities $T_{Y \rightarrow X}(\tau)$ and $T_{X \rightarrow Y}(\tau)$ will tell whether the process $Y$ influences $X$ more than vice-versa: if $T_{Y \rightarrow X} \geq T_{X \rightarrow Y}$ this is the case. Here the differential transfer entropy (DTE) from $Y$ to $X$

$\Delta T_{Y \rightarrow X} \stackrel{\text { def }}{=} T_{Y \rightarrow X}-T_{X \rightarrow Y}$

is used: when $\Delta T_{Y \rightarrow X}>0$ the dynamical influence $Y \rightarrow X$ prevails on the dynamical influence $X \rightarrow Y$.

The usefulness of $T_{Y \rightarrow X}$ in the study of the inter-scale dynamics of the magnetospheric turbulence is clear once the two time series $x(t)$ and $y(t)$ in (5) and (6) are two time series $\varepsilon_{\ell}(t)$ and $\varepsilon_{\ell^{\prime}}(t)$ describing the turbulent evolution at two different scales $\ell$ and $\ell^{\prime}$. Hence, the quantity

$$
\begin{aligned}
& T_{\ell \rightarrow \ell^{\prime}}(\tau)= \\
& =\sum_{\substack{\varepsilon_{\ell^{\prime}}(t) \\
\varepsilon_{\ell^{\prime}}(t-\tau) \\
\varepsilon_{\ell}(t-\tau)}} p\left(\varepsilon_{\ell^{\prime}}(t+\tau), \varepsilon_{\ell^{\prime}}(t), \varepsilon_{\ell}(t)\right) \log _{2}\left(\frac{p\left(\varepsilon_{\ell^{\prime}}(t+\tau) \mid \varepsilon_{\ell^{\prime}}(t), \varepsilon_{\ell}(t)\right)}{p\left(\varepsilon_{\ell^{\prime}}(t+\tau) \mid \varepsilon_{\ell^{\prime}}(t)\right)}\right)
\end{aligned}
$$

can be defined, and similarly $T_{\ell^{\prime} \rightarrow \ell}(\tau)$. Considering that in the phenomenon of direct cascade the larger structures determine the smaller ones, while in the case of inverse cascade the opposite takes place, one can use the DTE

$$
\Delta T_{\ell \rightarrow \ell^{\prime}}(\tau)=T_{\ell \rightarrow \ell^{\prime}}(\tau)-T_{\ell^{\prime} \rightarrow \ell}(\tau), \ell>\ell^{\prime}
$$

to determine the prevailing verse of cascade processes: when $\Delta T_{\ell \rightarrow \ell^{\prime}}>0$ the direct cascade from $\ell$ to $\ell^{\prime}<\ell$ is taking place, while if $\Delta T_{\ell \rightarrow \ell^{\prime}}<0$ an inverse one is at work.

It's important to say that analysing the time series collected along the POLAR trajectory and treating them as probabilistic evolutions we are not looking at the single direct or inverse cascade event of "eddies" crumbling or coalescing: while crossing the MC the spacecraft builds up $\boldsymbol{B}(t)$ visiting different points of the plasma flow. Different cascade events are then put together: the verse of the cascade indicated by the TEA will then be simply the statistically prevalent verse.

\subsection{The scale variables}

In order to single out the excitations at given scales, the time series $\boldsymbol{B}(t)$ is wavelet-transformed by using a Morlet wavelet as the mother wavelet of the continuous transform:

$$
\left\{\begin{array}{l}
\tilde{B}_{\ell}^{(i)}(t)=\frac{1}{\sqrt{\ell}} \int_{I} B_{i}\left(t^{\prime}\right) \psi^{*}\left(\frac{t^{\prime}-t}{\ell}\right) d t^{\prime}, \\
\psi(x)=\pi^{-\frac{1}{4}} \exp \left(i \omega_{0} x-\frac{1}{2} x^{2}\right) .
\end{array}\right.
$$

where $I$ is the time interval on which the series $\boldsymbol{B}(t)$ is defined. Then the square modulus of this complex time series $\tilde{B}_{\ell}^{(i)}(t)$ is used:

$\varepsilon_{\ell}^{(i)}(t) \stackrel{\text { def }}{=}\left|\tilde{B}_{\ell}^{(i)}(t)\right|^{2}$.

All the parameter values chosen for the analysis are justified in Appendix A. Once the definition (10) is given, the quantity $T_{\ell \rightarrow \ell^{\prime}}^{(i)}$ is calculated by assuming the evolutions $\varepsilon_{\ell}^{(i)}$ and $\varepsilon_{\ell^{\prime}}^{(i)}$ to be describing stationary processes, and evaluating the probabilities as the normalized histograms. These probabilities are directly inserted in (7).

\subsection{Adjacent scale TEA}

The transfer entropies $T_{\ell \rightarrow \ell^{\prime}}^{(i)}$ and $T_{\ell^{\prime} \rightarrow \ell}^{(i)}$ can be calculated for any couple of scales $\left(\ell, \ell^{\prime}\right)$, but one choice seems particularly meaningful for the study of cascade processes. Since in the traditional picture of turbulence the most important interscale coupling giving rise to cascade processes takes place between fluctuations at two nearby scales, a sensible choice for the couple $\left(\ell, \ell^{\prime}\right)$ is $\ell^{\prime}=\ell-d \ell$. It is likely that also other couplings may take place, between largely different scales too. This will be the object of future studies.

Due to the numerical implementation of the continuous wavelet transform (9), the difference $d \ell$ will rather be intended as

$d \ell_{n}=\ell_{n+1}-\ell_{n}$,

being $\left\{\ell_{n}\right\}$ the discrete sequence of scales adopted in the discretization of the continuous transform (9). In Appendix A the expression for $\left\{\ell_{n}\right\}$ is given together with $d \ell_{n}$ in (A4) and (A5).

The quantities $T_{\ell \rightarrow \ell-d \ell}^{(i)}(\tau)$ and $T_{\ell-d \ell \rightarrow \ell}^{(i)}(\tau)$ have been constructed by applying (7) holding the delay $\tau$ fixed as twice the sampling time $d t=0.12 \mathrm{~s}$. This choice of $\tau=2 d t$ corresponds to analysing the dynamics on the shortest possible time within which an interaction may be captured by studying the given time series. Once we've done so, the differential entropy

$\Delta T_{\ell \rightarrow \ell-d \ell}^{(i)}=T_{\ell \rightarrow \ell-d \ell}^{(i)}(\tau)-T_{\ell-d \ell \rightarrow \ell}^{(i)}(\tau)$

depends only on the scale $\ell$, and can be studied as a function $\Delta T_{\ell \rightarrow \ell-d \ell}^{(i)}(\ell)$. Such an analysis is expected to define those intervals of scales in which direct cascade processes prevail and those in which the opposite takes place. The anisotropy described in Yordanova et al. (2005) is expected to possibly give different behaviours of $\Delta T_{\ell \rightarrow \ell-d \ell}^{(i)}(\ell)$ for $i=0$ (axial component) or $i=1,2$ (poloidal components).

In order to find out synthetically what the TEA analysis may teach about the magnetic turbulence in the cusp, $\Delta T_{\ell \rightarrow \ell-d \ell}^{(i)}$ will be presented rather as a function of the 

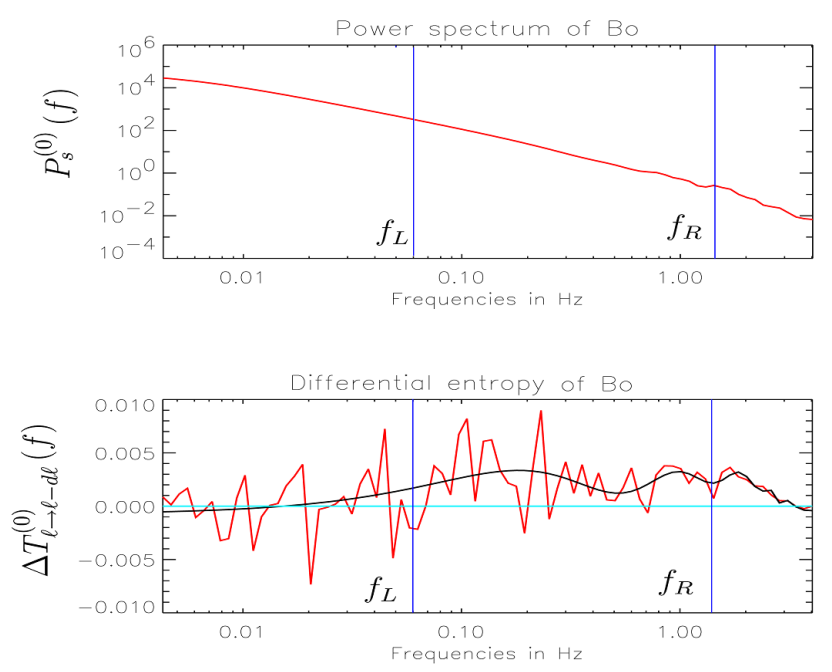

Fig. 3. Comparison between the power spectrum $P_{S}^{(0)}(f)$ of the axial component $B_{0}$ (top) and the DTE $\Delta T_{\ell \rightarrow \ell-d \ell}^{(0)}(f)$ (bottom). $f_{L}$ and $f_{R}$ are the limits of the turbulent cascade determined in Yordanova et al. (2005). In the bottom picture the red line is $\Delta T_{\ell \rightarrow \ell-d \ell}^{(0)}(f)$ from the real data, while the black smooth line is its polynomial fit $\mathcal{G}_{20}^{(0)}(f)$. In the spectrum, note a local (extremely weak) hump at $\sim 0.2 \mathrm{~Hz}$, local bumps at $\sim 0.8 \mathrm{~Hz}, \sim 1 \mathrm{~Hz}$ and $f_{R}$ (the latter is the most visible feature), and three slight bumps at higher frequencies. In the fitted curve $\mathcal{G}_{20}^{(0)}(f)$ note the local maxima at $\sim 0.2 \mathrm{~Hz}, \sim 1 \mathrm{~Hz}$ and $\sim 2 \mathrm{~Hz}$, and the local minima between $0.5 \mathrm{~Hz}$ and $0.6 \mathrm{~Hz}$, and at about $f_{R}$.

frequency

$f(\ell)=\frac{\omega_{0}+\sqrt{\omega_{0}^{2}+2}}{\ell}$

of the Fourier component corresponding to the time-scale $\ell$ of the wavelet analysis. Hence $\Delta T_{\ell \rightarrow \ell-d \ell}^{(i)}(f)$ will be compared with the Fourier power spectrum $P_{s}^{(i)}(f)$ of the $i$-th component of $\boldsymbol{B}(t)$, so that the physical features of $\Delta T_{\ell \rightarrow \ell-d \ell}^{(i)}(f)$ will be straightforwardly compared with those already interpreted in Yordanova et al. (2005).

\subsection{Results}

The time series under exam was studied by Yordanova et al. (2004) and in Yordanova (2005) in terms of its multifractal properties as far as the magnetic energy $|\boldsymbol{B}|^{2}$ was concerned. In Yordanova et al. (2005) the spectral properties of the time series $B_{0}(t), B_{1}(t)$ and $B_{2}(t)$ were analysed, together with the PDFs as appearing at different (time) scales. The results found there appear to be in reasonable agreement with the picture described by Chang and his co-authors in Chang et al. (2004): the spacecraft is crossing a "gas" of filamentary current "structures" interacting with each other via coalescence and fragmentation, giving rise to inverse and di-
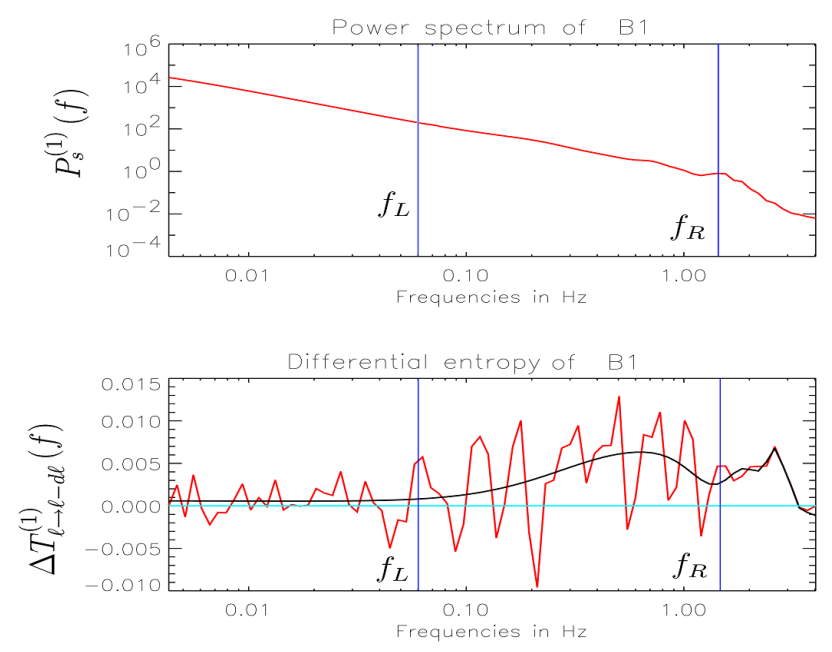

Fig. 4. Comparison between the power spectrum $P_{S}^{(1)}(f)$ of the first poloidal component $B_{1}$ (top) and the DTE $\Delta T_{\ell \rightarrow \ell-d \ell}^{(1)}(f)$ (bottom). $f_{L}$ and $f_{R}$ are the limits of the turbulent cascade determined in Yordanova et al. (2005). In the bottom picture the red line is $\Delta T_{\ell \rightarrow \ell-d \ell}^{(1)}(f)$ from the real data, while the black smooth line is its polynomial fit $\mathcal{G}_{20}^{(1)}(f)$. In the spectrum, note a local (extremely weak) hump at $\sim 0.2 \mathrm{~Hz}$, local bumps at $\sim 0.8 \mathrm{~Hz}, \sim 1 \mathrm{~Hz}$ and $f_{R}$ (the latter is the most visible feature). Three slight bumps at higher frequencies. In the fitted curve $\mathcal{G}_{20}^{(0)}(f)$ note the local maxima at $\sim 0.7 \mathrm{~Hz}, \sim 2 \mathrm{~Hz}$ and $\sim 3 \mathrm{~Hz}$ and the local minima slightly before $f_{R}$ and between $2 \mathrm{~Hz}$ and $3 \mathrm{~Hz}$.

rect cascade events respectively. The result presented here as obtained from the TEA will confirm and enrich that picture with the indication of the statistically prevalent verse of the fragmentation-coalescence interactions.

The results have been checked via a surrogate data test, described in 2.3.2.

\subsubsection{Quantities from real data}

The Fourier spectra of the components of $\boldsymbol{B}(t)$ were calculated in Yordanova et al. (2005) as power laws with two "breaks" at the frequencies

$$
f_{L} \sim e q 0.06 \mathrm{~Hz}, f_{R} \in[1 \mathrm{~Hz}, 2 \mathrm{~Hz}] .
$$

The frequencies $f_{L}$ and $f_{R}$ may be interpreted as delimiting the inertial range of the turbulent cascade: according to this interpretation, the energy would be injected at the scale $\ell\left(f_{L}\right)$ and cascade down to the scale $\ell\left(f_{R}\right)$, where it is dissipated. The frequency $f_{R}$ is considered as the cusp ion cyclotron frequency, where $f_{L}$ is rather assimilated to the Solar Wind ion cyclotron frequency, so to say that the harmonic components of $\boldsymbol{B}(t)$ with $f<f_{L}$ are governed by the flux of the Solar Wind. This Solar Wind driving is mainly affecting the axial component $B_{0}(t)$.

The behaviour of $\Delta T_{\ell \rightarrow \ell-d \ell}^{(i)}(f)$ is presented in the Figures from 3 to 5, while Fig. 6 refers to the excitations in the 

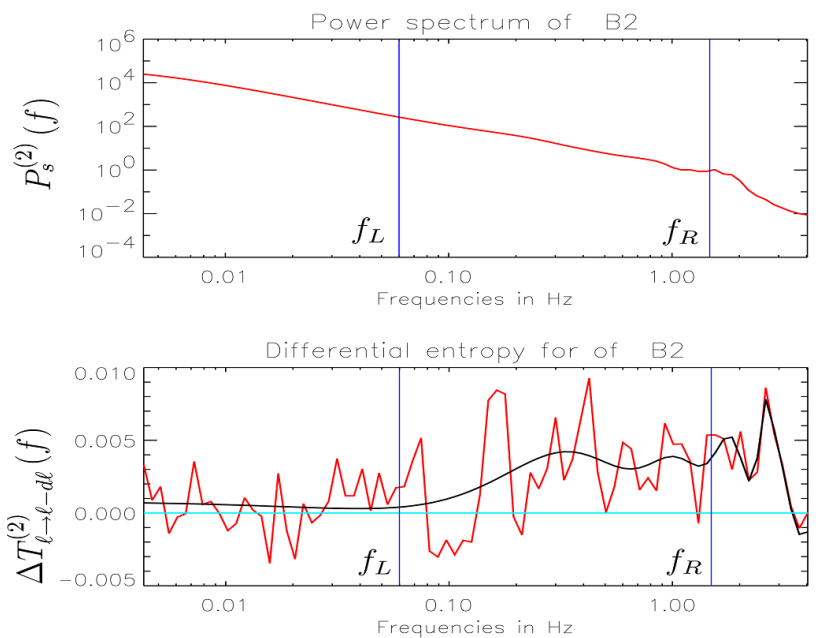

Fig. 5. Comparison between the power spectrum $P_{S}^{(2)}(f)$ of the second poloidal component $B_{2}$ (top) and the DTE $\Delta T_{\ell \rightarrow \ell-d \ell}^{(2)}(f)$ (bottom). $f_{L}$ and $f_{R}$ are the limits of the turbulent cascade determined in Yordanova et al. (2005). In the bottom picture the red line is $\Delta T_{\ell \rightarrow \ell-d \ell}^{(2)}(f)$ from the real data, while the black smooth line is its polynomial fit $\mathcal{G}_{20}^{(2)}(f)$. In the spectrum, note a local (extremely weak) hump at $\sim 0.2 \mathrm{~Hz}$, local bumps at $\sim 0.9 \mathrm{~Hz}$ and $f_{R}$ (the latter is the most visible feature). In the fitted curve $\mathcal{G}_{20}^{(0)}(f)$ note the local maxima at $\sim 0.3 \mathrm{~Hz}, \sim 1 \mathrm{~Hz}$ and $\sim 2 \mathrm{~Hz}$ and $3 \mathrm{~Hz}$, and the local minima at $\sim 0.6 \mathrm{~Hz}$, slightly before $f_{R}$ and slightly after $2 \mathrm{~Hz}$.

poloidal intensity $B_{n}$

$B_{n}=\sqrt{B_{1}^{2}+B_{2}^{2}}$.

Looking at the behaviours of $\Delta T_{\ell \rightarrow \ell-d \ell}^{(i)}$ with $f$ (red plots in the bottom panels of Figs. from 3 to 6) it is difficult to distinguish a tendency or even to establish clearly for which frequencies the sign of $\Delta T_{\ell \rightarrow \ell-d \ell}^{(i)}$ points towards a direct or an inverse cascade. All is possible to state at first glance is that $\Delta T_{\ell \rightarrow \ell-d \ell}^{(i)}$ goes to zero well outside the interval of frequencies $\left[f_{L}, f_{R}\right]$

$$
f \ll f_{L}, f \gg f_{R} \Rightarrow \Delta T_{\ell \rightarrow \ell-d \ell}^{(i)}(f) \sim e q 0
$$

and that it oscillates around zero without a well apparent sign. The first physical conclusion should be that events of coalescence and fragmentation of the turbulent structures are both present and probably mixed at all the scales.

In order to target more clearly some "theoretical" behaviour of $\Delta T_{\ell \rightarrow \ell-d \ell}^{(i)}(f)$, it is then tried to draw a "fitting curve" through the values calculated from the data (black plot in the bottom panels of Figs. from 3 to 6). An $N$ degree polynomial fit

$\mathcal{G}_{N}^{(i)}(f)=\sum_{k=0}^{N} g_{k}^{(i)} f^{k}$
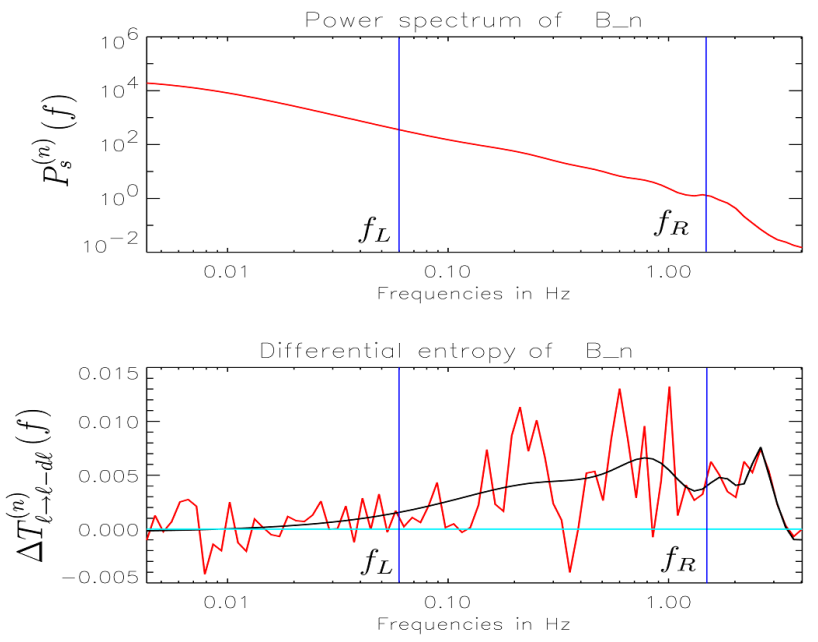

Fig. 6. Comparison between the power spectrum $P_{s}^{(n)}(f)$ of the poloidal intensity $B_{n}$ (top) and the DTE $\Delta T_{\ell \rightarrow \ell-d \ell}^{(n)}(f)$ (bottom). $f_{L}$ and $f_{R}$ are the limits of the turbulent cascade determined in Yordanova et al. (2005). In the bottom picture the red line is $\Delta T_{\ell \rightarrow \ell-d \ell}^{(n)}(f)$ from the real data, while the black smooth line is its polynomial fit, $\mathcal{G}_{20}^{(n)}(f)$. In the spectrum, note the local (extremely weak) hump at $\sim 0.2 \mathrm{~Hz}$, local bumps at $\sim 0.8 \mathrm{~Hz}, \sim 1 \mathrm{~Hz}$ and $f_{R}$ (the latter is the most visible feature). In the fitted curve $\mathcal{G}_{20}^{(0)}(f)$ note the local hump at $\sim 0.2 \mathrm{~Hz}$, the local maxima at $\sim 0.8 \mathrm{~Hz}$, slightly after $f_{R}$ and slightly before $3 \mathrm{~Hz}$, and the local minima slightly before $f_{R}$ and at $\sim 2 \mathrm{~Hz}$.

is hence superimposed to the values of $\Delta T_{\ell \rightarrow \ell-d \ell}^{(i)}(f)$. Then, the physical conclusions are drawn from the analysis of the smooth, more readable plot $\mathcal{G}_{N}^{(i)}(f)$. The degree $N$ is fixed as $N=20$, because this is the best value to satisfy the condition (15)

$f \ll f_{L}, f \gg f_{R} \Rightarrow \mathcal{G}_{20}^{(i)}(f) \sim e q 0$.

The details of the comparisons between the behaviour of the $\mathcal{G}_{20}^{(i)} \mathrm{s}$ and the spectra are reported completely in the captions of the figures.

In general, it can be stated that the sign of $\mathcal{G}_{20}^{(i)}(f)$ is rather well defined in the inertial interval $\left[f_{L}, f_{R}\right]$, where indeed it is pointing towards positive $\Delta T_{\ell \rightarrow \ell-d \ell}^{(i)}(f)$, i.e. direct cascade:

$f_{L}<f<f_{R} \Rightarrow \mathcal{G}_{20}^{(i)}(f)>0$.

Comparing the plots of $P_{s}^{(i)}(f)$ and $\mathcal{G}_{20}^{(i)}(f)$ one discovers that the stationary points of $\mathcal{G}_{20}^{(i)}(f)$ correspond roughly to (more or less weak) local "bumps" on $P_{s}^{(i)}(f)$, denoting the correspondence between local maxima of the differential transfer entropy and spectral features. A minimum of $\mathcal{G}_{20}^{(i)}(f)$ is located slightly before the "break" frequency $f_{R}$.

As far as the anisotropy between the axial and the poloidal directions is concerned, the most noticeable difference is that 

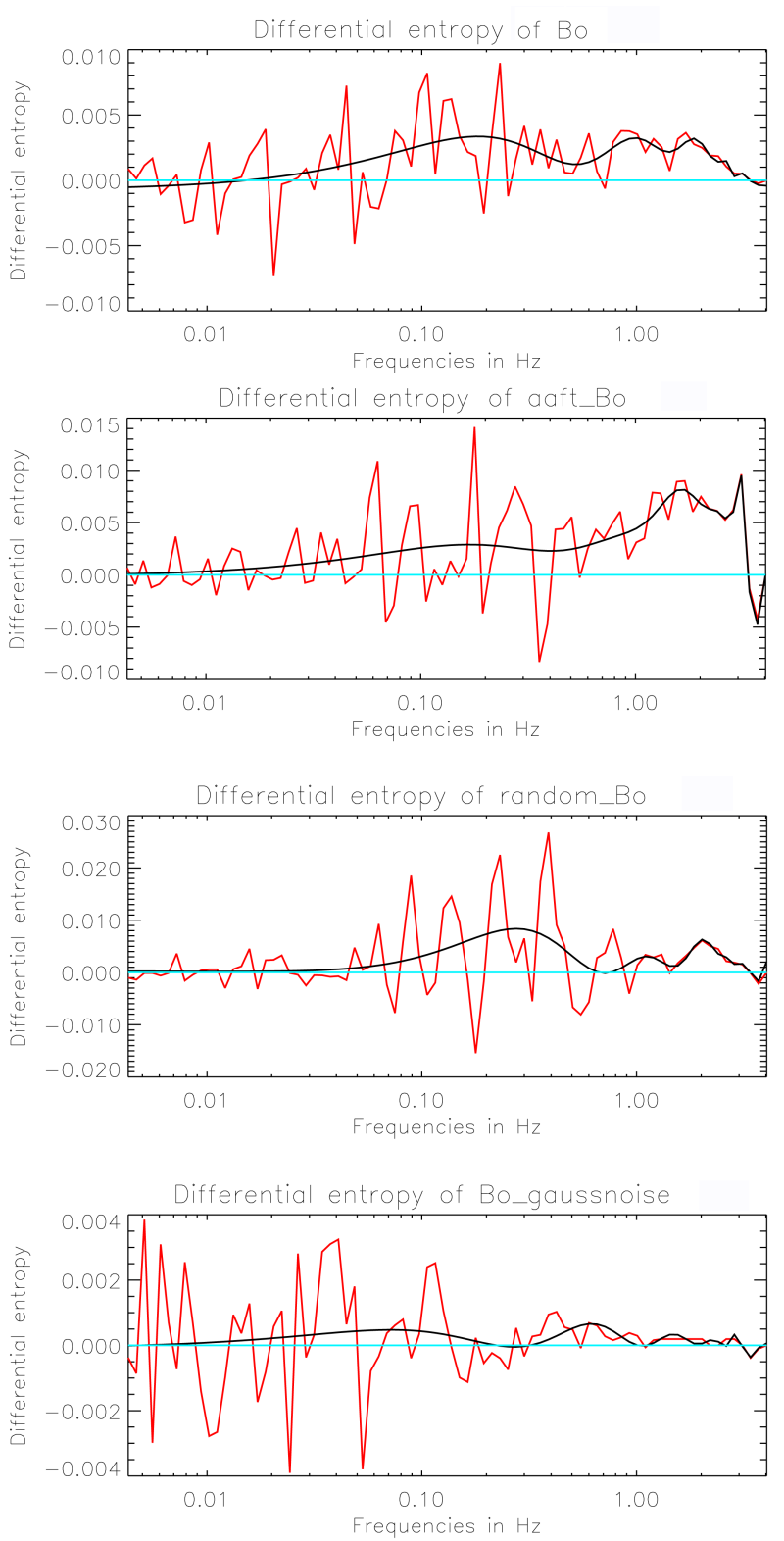

Fig. 7. Comparison of $\Delta T_{\ell \rightarrow \ell-d \ell}^{(0)}$ and $\mathcal{G}_{20}^{(0)}$ as calculated from the time series $B_{0}(t)$ and from the surrogate data associated to it. From top to bottom: real time series, AAFT surrogate data (series "aaft_B0"), phase randomized surrogate data (series "random_B0") and a Gaussian noise (series "B0_gaussnoise").

$\mathcal{G}_{20}^{(0)}$ is smaller than $\mathcal{G}_{20}^{(1,2)}$ and $\mathcal{G}_{20}^{(n)}$, so that along the poloidal plane the "direct cascade" events are more importantly prevalent than along $\hat{e}_{/ /}$. Another difference between $\mathcal{G}_{20}^{(0)}$ and $\mathcal{G}_{20}^{(1,2, n)}$ is that $\mathcal{G}_{20}^{(1,2, n)}$ have an enhancement at frequencies higher than $f_{R}$, going to zero then, this increase being much smaller for $\mathcal{G}_{20}^{(0)}$.
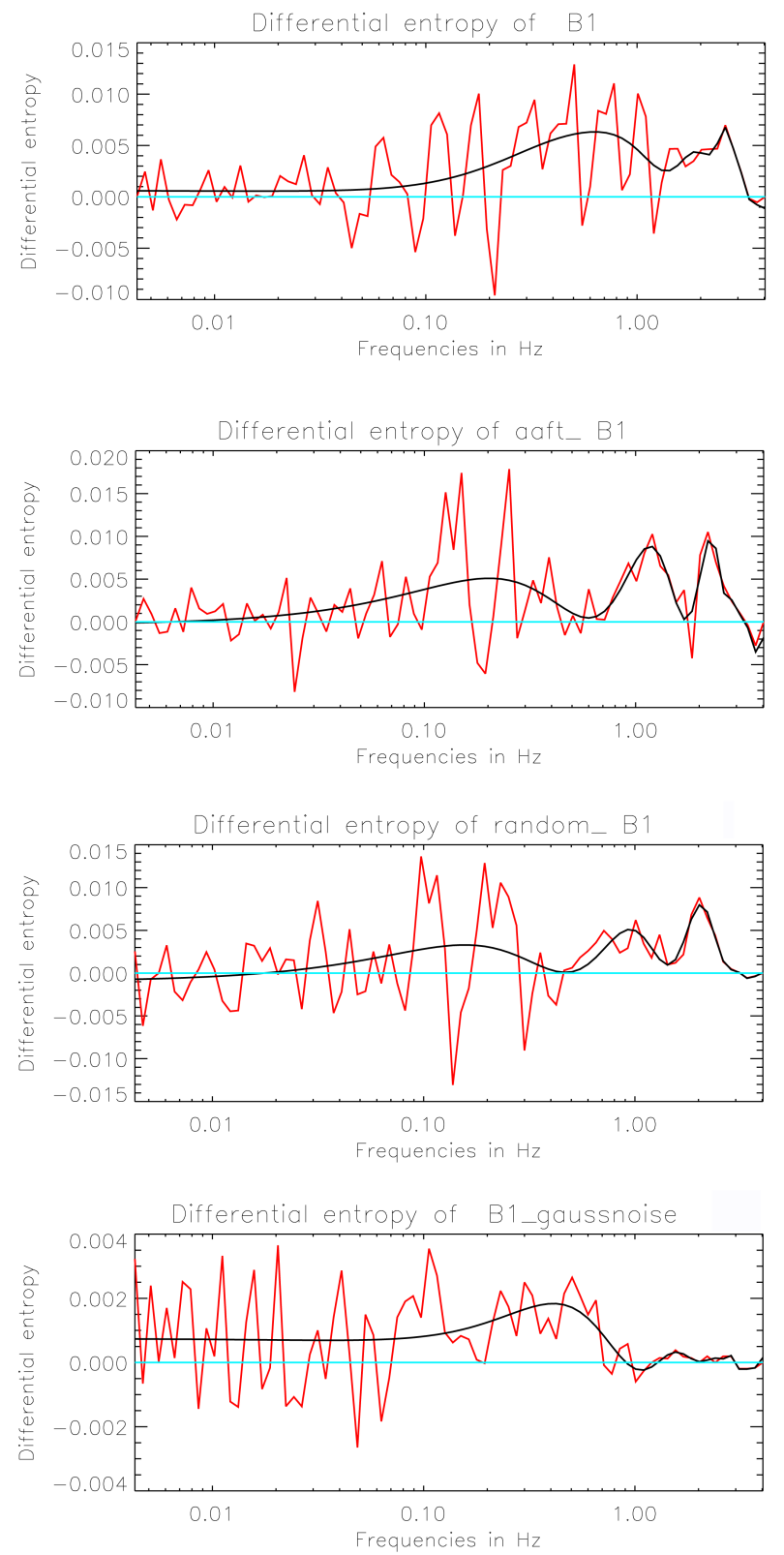

Fig. 8. Comparison of $\Delta T_{\ell \rightarrow \ell-d \ell}^{(1)}$ and $\mathcal{G}_{20}^{(1)}$ as calculated from the time series $B_{1}(t)$ and from the surrogate data associated to it. From top to bottom: real time series, AAFT surrogate data (series "aaft_B1"), phase randomized surrogate data (series "random_B1") and a Gaussian noise (series "B1_gaussnoise").

\subsubsection{Surrogate data test}

In order to check how reliable these results may be considered, a surrogate data test for the nonlinearity of the dynamics producing them may be used. Indeed, nonlinear couplings should be the origin of cascades and coalescence events.

The results of the surrogate data test are presented in Figures from 7 to 9 . 
For every time series analysed one may produce the surrogate data described in Theiler et al. (1992), in particular the phase-randomized surrogate data (indicated in the Figures as "random") and the Amplitude Adjusted Fourier Transform surrogate data (indicated in the Figures as 'aaft'). The phase randomized data are constructed as the Gaussian linear process with the same power spectrum of the original real data, while the 'aaft' data are supposed to mimic a process obtained from the linear Gaussian one with the same power spectrum of the original real data via a non-linear transformation. We have also produced pure Gaussian noises without any relationship with the real data.

The results of the surrogate data test is indicating that the quantities $\Delta T_{\ell \rightarrow \ell-d \ell}^{(i)}$ and $\mathcal{G}_{20}^{(i)}$ of the real data are not very much different from those obtained from surrogate data, apart in the Gaussian noise case. On one hand, considering that the "essentially linear" time series constructed via the "random" or via the "aaft" procedures should have zero $\Delta T_{\ell \rightarrow \ell-d \ell}^{(i)}$ everywhere, this result should be interpreted in stating that $\Delta T_{\ell \rightarrow \ell-d \ell}^{(i)}$ and $\mathcal{G}_{20}^{(i)}$ are not meaningfully different from zero, in general, so that one might conclude

$$
\Delta T_{\ell \rightarrow \ell-d \ell}^{(i)}(f) \sim e q 0, \mathcal{G}_{20}^{(i)}(f) \sim e q 0 \forall f \in[0.006 \mathrm{~Hz}, 4 \mathrm{~Hz}]
$$

This would point towards an equivalence between the occurrence of the direct and inverse cascade events registred during the flight of the spacecraft.

On the other hand, since both the "aaft" as well as the phase-randomized "random" surrogate data have the same power spectrum as the real ones, and since $\mathcal{G}_{20}^{(i)}(f)$ is seen to have a rather good correlation with the $P_{S}^{(i)}(f)$ features, there is no surprise in the apparently deluding results of Figs. from 7 to 9 .

\section{Discussion}

The Earth's MC has always been known as a region of highly turbulent plasma. The presence of plasma turbulence produces a state of turbulence in the local magnetic field as well, due to the strict coupling between matter and field in a conducting fluid. The turbulent magnetic field is then expected to show those non-linear phenomena of turbulent systems as energy cascades due to inter-scale couplings.

In principle, processes of fragmentation of magnetic structures into smaller ones, and coalescence of magnetic structures forming bigger size excitations are both possible. In the present paper the transfer entropy introduced in Schreiber (2000) has been used to establish the existence of a prevalent sign in these cascade processes. The quantity studied is the differential transfer entropy $\Delta T_{\ell \rightarrow \ell-d \ell}^{(i)}$ telling how much the dynamical driving of the $\ell$-sized structures onto the $(\ell-d \ell)$ sized structures is stronger than the opposite one. The causal
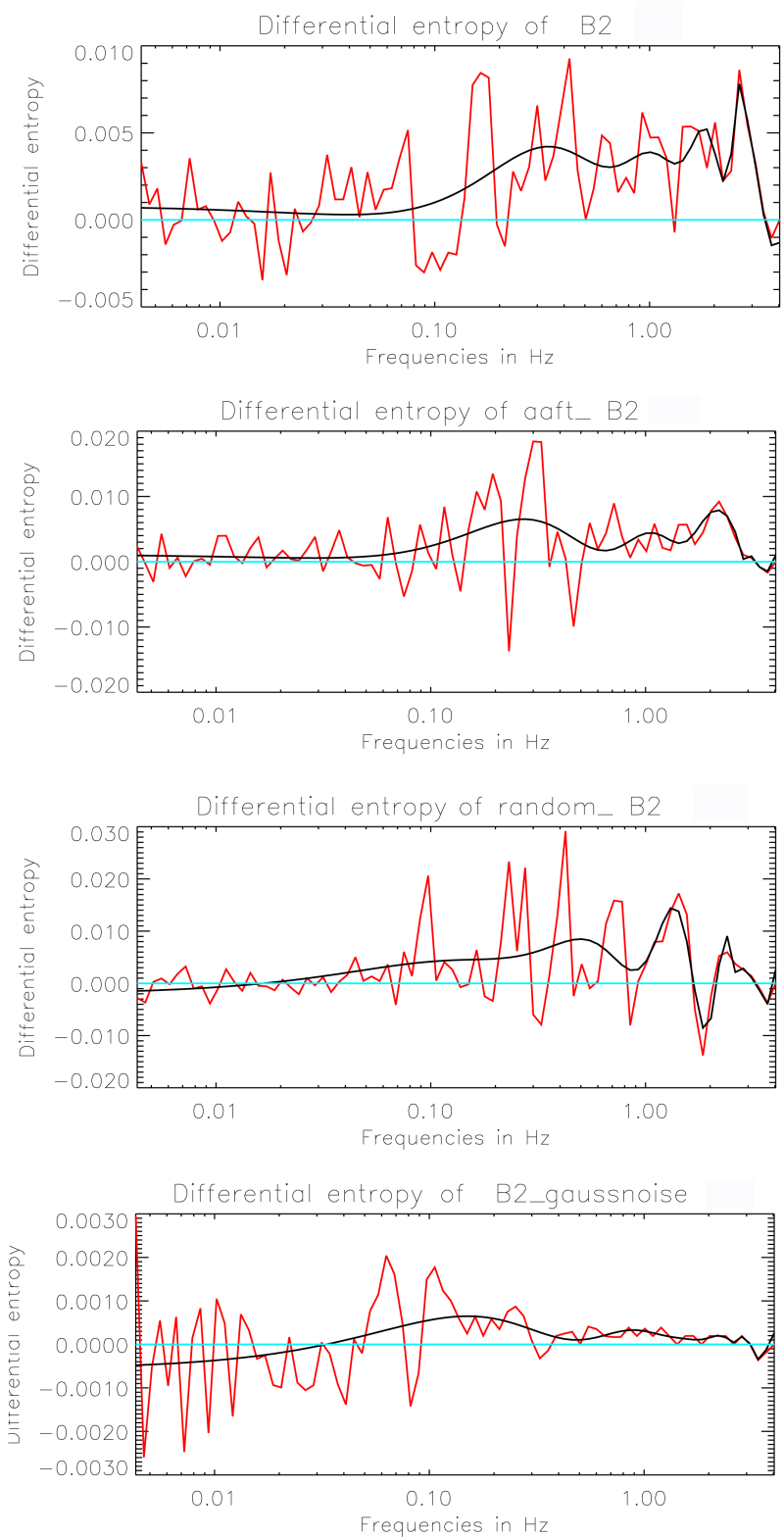

Fig. 9. Comparison of $\Delta T_{\ell \rightarrow \ell-d \ell}^{(2)}$ and $\mathcal{G}_{20}^{(2)}$ as calculated from the time series $B_{2}(t)$ and from the surrogate data associated to it. From top to bottom: real time series, AAFT surrogate data (series "aaft_B2"), phase randomized surrogate data (series "random_B2") and a Gaussian noise (series "B2_gaussnoise").

delay considered is $\tau=0.24 \mathrm{~s}$, as long as twice the sampling time of the data.

The plot of $\Delta T_{\ell \rightarrow \ell-d \ell}^{(i)}$ as a function of the frequency is indicating the existence of a frequency interval within which a direct cascade regime should be prevalent. This frequency interval is slightly wider than the inertial interval obtained in Yordanova et al. (2005) studying the power spectrum of the magnetic axial and poloidal excitations. The plots 
of $\Delta T_{\ell \rightarrow \ell-d \ell}^{(i)}(f)$ and its polynomial fit $\mathcal{G}_{20}^{(i)}(f)$ show behaviours rather correlated with the (weak) features appearing in the power spectrum $P_{s}^{(i)}(f)$. This correlation between the DTEs and the power spectra is showing up in all the components of $\boldsymbol{B}$, even if the inter-scale coupling taking place within the causal delay considered here is stronger along the poloidal directions.

The surrogate data test used criticizes strongly the reliability of the values $\Delta T_{\ell \rightarrow \ell-d \ell}^{(i)}$ found here, and in 2.3.2.this has been used to guess (15). However, strictly speaking, the fact that $\Delta T_{\ell \rightarrow \ell-d \ell}^{(i)}$ and $\mathcal{G}_{20}^{(i)}$ for real and surrogate data are not very much different does not mean that (15) is satisfied. Instead, it means that the approach based on the wavelet transform and the transfer entropy notion is not sufficient to conclude whether we are dealing with the cascade process or linear Gaussian process (random) or nonlinearly transformed Gaussian process (aaft). It is worth reminding that the occurrence of dual cascades, i.e. regimes in which direct and inverse cascade events are well possible, has been repeatedly predicted in the literature about magnetic turbulence in space plasmas. In particular, one may quote the works by Chang (see Chang et al. (2004) and those quoted in Yordanova et al. (2005)), claiming for a regime of very complex interactions among coarse-grained field and current configurations; the paper Savin et al. (2004), in which single events of direct and inverse cascades are targeted in scalograms; the plasma simulations International School ASSE (2006) shown by Shukla in his invited paper in L'Aquila for the Summer School ASSE 2006.

Some more final comments should be considered.

First of all, the results presented here are still very preliminary ones, obtained via still developing tools (the application of transfer entropy to wavelet-analysed geospace data is rather new, as far as the Authors are aware of).

Continuous wavelet transform, used in our analysis, is a redundant transform, which means that the wavelet transform coefficients are correlated. This property might affect the transfer entropy calculations. We plan to use the discrete wavelet transform but this can be done at the expense of frequency (scale) resolution.

The data collected here are obtained as measurements along the satellite trajectory, as written in (1): this means that we are using the identification of time and space statistics of the turbulent field, which might be a naïve point of view. Many-point simultaneous measurements should be used in future investigations, to give a more rigorous and reliable idea of the space configuration of the field. In the present paper one cannot claim to be calculating the proper ensemble statistics defining the transfer entropies theoretically. It's only possible to interpret the indications of $\Delta T_{\ell \rightarrow \ell-d \ell}^{(i)}(f)$ as valid on the set of cascade events encountered by the spacecraft along its trajectory.

These are sample results obtained with very few statistics: we are actually facing a highly variable system (the magneto- plasma turbulence in the Earth's MC) with a simple case study.

One should mention that the Earth's MC is one the most highly variable and complicated region of the geospace: in order to refine the mathematical tools adopted here it will be necessary to try them on the simpler cases of synthetic turbulence data (in which one completely knows the verse of the "cascades" occurring) and of real data collected in less complicated, and more well known, regions of the Heliogeospace.

Last but not least, all these calculations should be repeated for many values of the causal delay $\tau$. This should allow to explore the entropies relative to processes occurring in a lapse of time equal to $\tau_{\ell}$, the typical time of the energy transfer from $\ell$ to $\ell-d \ell$.

\section{Appendix A}

\section{Choosing the wavelet analysis parameters}

The wavelet analysis technique is extensively used in the study of fluid turbulence and irregular media Farge et al. (1999).

The continuous wavelet transform coefficients are time series of the same length and sampling of the original one, for all the scales considered. The definition of the transform is formula (9). The Morlet function is chosen as mother wavelet because it is very good for the time and frequency resolution, matching the minimum uncertainty condition. Being $\sigma_{f}$ and $\sigma_{t}$ the frequency and time spread respectively, one has indeed:

$$
\omega_{0}^{2} \gg 1 \Rightarrow \sigma_{f} \sigma_{t}=\frac{1}{4 \pi} \text {. }
$$

Here we have chosen $\omega_{0}=6$, so that $\psi(x)$ turns out to fulfill the admissibility condition Farge (1992).

In principle the transform (9) allows for a continuous spectrum of the scales $\ell$, but a discretisation is done for pursuing the numerical implementation of it Torrence and Compo (1998). The scales admitted are given by the collection

$\ell_{n}=2^{\frac{n}{v}} \ell_{\min }, n \in \mathbb{N}$,

being $V$ a positive integer referred to as number of voices, so that $\ell_{n+V}=2 \ell_{n}$. If $V$ is chosen suitably the wavelet constructed are quasi-orthogonal, and select quasi-independent scales. Some mathematical results Daubechies (1992) and Mallat (1998) indicate that with $V \geq 4$ this property is fulfilled. Here we have chosen $V=8$.

The minimum scale $\ell_{\min }$ is fixed as twice the sampling time

$\ell_{\min }=2 d t=0.24 \mathrm{~s}$.

With our parameters one obtains the scale sequence

$\ell_{n}=2^{\frac{n}{8}} \cdot 0.24 \mathrm{~s}, n \in \mathbb{N}$. 
The minimal difference of scales distinguished is then:

$d \ell(n)=\ell_{n+1}-\ell_{n}=2^{\frac{n}{8}}\left(2^{\frac{1}{8}}-1\right) \cdot 0.24 \mathrm{~s}=2^{\frac{n}{8}} \times 0.02 \mathrm{~s}$,

This will be the $d \ell$ separating the two adjacent scales in our analysis. It grows exponentially with $n$.

Acknowledgements. The Authors would like to thank C. Russel, PI of the experiment MFE on board of the POLAR mission, for making the data available on the web. They also would like to thank B. Popielawska for her helpful discussions.

MM's and EY's work was supported by the European Community's Human Potential Programme under contract HPRN-CT-200100314, "Turbulent Boundary Layers in geospace Plasmas".

Edited by: P.-L. Sulem

Reviewed by: two referees

\section{References}

Frisch, U.: Turbulence, the legacy of A. N. Kolmogorov, Cambridge University Press, 1995.

Kraichnan, R. H. and Montgomery, D.: Two-dimensional turbulence, Reports Progr. Phys., 43, 547-619, 1980.

Savin, S., Zelenyi, L., Romanov, S., Standahl, I., Pickett, J., Rauch, J. L., Amata, E., Avanov, L., Blecki, J., Budnik, E., Büchner, J., Cattell, C., Consolini, G., Fedder, J., Fuselier, S., Kawano, H., Klimov, S., Korepanov, V., Lagoutte, D., Marcucci, F., Mogilevsky, M. Nemecek,, Z., Nikutowski, B., Nozdrachev, M., Parrot, M., Rauch, J. L., Romanov, V., Romantsova, T., Russell, C. T., Safrankova, J., Sauvaud, J. A., A. Skalsky, Smirnov, V., Stasiewicz, K., Trotignon, J. G., and Yarmolaev, Yu.: Magnetosheath-cusp interface, Ann. Geophys., 22, 183 212, 2004, http://www.ann-geophys.net/22/183/2004/.

Schreiber, T.: Measuring Information Transfer, Phys. Rev. Lett., 85, 461, 2000.
Keiser, A. and Schreiber, T.: Information transfer in continuous processes, Physica D, 166, 43-62, 2002.

Haken, H.: Synergetics, Springer-Verlag (Berlin, Heidelberg, New York, Tokyo), 1983.

Chang, T., Tam, S. W. Y., and Wu, C.-C.: Complexity induced anisotropic bimodal intermittent turbulence in space plasmas, Physics of Plasmas, 11(4), 1287, 2004.

Russel, C. T., Snare, R. C., Means, J. D., Pierce, D., Dearborn, D., Larson, M., Barr, G., and Le, G.: The GGS/Polar magnetic field investigations, Space Sci. Rev., 71, 563-582, 1995.

Yordanova, E., Bergman, J., Consolini, G., Kretzschmar, M., Materassi, M., Popielawska, B., Roca-Sogorb, M., Stasiewicz, K., and Wernik, A. W.: Anisotropic scaling features and complexity in magnetospheric-cusp: a case study, Nonlin. Processes Geophys., 13, 1-9, 2005.

Yordanova, E., Grzesiak, M., Wernik, A. W., Popielawska, B., and Stasiewicz, K.: Multifractal structure of turbulence in the magnetospheric cusp, Ann. Geophys., 22, 2431-2440, 2004, http://www.ann-geophys.net/22/2431/2004/.

Yordanova, E.: Scaling behaviour of Turbulence in the Polar cusp, thesis submitted for the degree of Doctor of Physics, Space Research Centre, Polish Academy of Science, Warsaw, 2005.

Theiler, J., Eubank, S., Longtin, A., Galdrikian, B., and Doyne Farmer, J.: Testing for nonlinearity in time series: the method of surrogate data, Physica D, 58, 77-94, 1992.

International School ASSE 2006. Aquila INFN, Rome, Italy, 11-15 September 2006, Nonlinear Processes in Space Plasmas (Tutorial Lecture).

Farge, M., Kevlahan, N. K.-N., Perrier, V., and Schneider, K.: Turbulence analysis, modeling and computing using wavelets, in: Wavelets in physics, edited by: van den Berg, J. C., Cambridge University Press, 1999.

Farge, M.: Wavelet transforms and their application to turbulence, Annu. Rev. Fluid. Mech., 24, 395-457, 1992.

Torrence, C. and Compo, G. P.: A practical guide to wavelet analysis, Bull. Am. Meteorol. Soc., 79(1), 61-78, 1998.

Daubechies, I.: Ten lectures on wavelets, SIAM, Philadelphia, 1992.

Mallat, S.: A wavelet tour of signal processing, Academic Press, 1998. 